\title{
Tecnologias educacionais desenvolvidas para o cuidado ao paciente diabético: revisão integrativa da literatura
}

\author{
Educational technologies developed for diabetic patient care: integrative literature review \\ Tecnologías educativas desarrolladas para el cuidado del paciente diabético: revisión \\ integrativa de la literatura
}

José Vinícius de Souza ${ }^{1 *}$, Mateus Andrade Ferreira ${ }^{2}$, José Isaac Alves de Andrade², Anna Valéria Duarte Calixto ${ }^{1}$, Rafaelle Cavalcante de Lira ${ }^{1}$.

\section{RESUMO}

Objetivo: Realizar um levantamento bibliográfico sobre as principais tecnologias educacionais utilizadas no cuidado com pacientes com Diabetes Mellitus. Métodos: Trata-se de uma revisão integrativa de literatura retrospectiva com caráter descritivo-exploratório realizada nas bases de dados LILACS, Web of Science, PUBMED e SCIELO, por meio do cruzamento dos descritores "Diabetes Mellitus", "Complicações do Diabetes", "Tecnologia Educacional", "Estudo de Validação" e "Jogos e Brinquedos", nos idiomas português, inglês e espanhol. Resultados: A amostra final foi constituída de 20 artigos que emergiram duas categorias temáticas: Tipos de tecnologias cuidativo-educacionais utilizadas na promoção do autocuidado em diabéticos e Interface entre aprendizagem significativa e as dificuldades na implementação das tecnologias cuidativoeducacionais. O cenário atual de produção de educação em saúde utiliza, sobretudo, como recurso, as mídias digitais como games, porém ainda conta com a participação significativa de ferramentas impressas e físicas. É notória a necessidade de adaptação quanto ao conteúdo e linguagem a fim de atender à demanda sociocultural do público abordado. Considerações Finais: A produção de tecnologias educacionais para diabéticos está, em sua maioria, pautada em mídias digitais. Notou-se a carência de jogos de tabuleiro para o público em questão.

Palavras-chave: Diabetes mellitus, Complicações do diabetes, Tecnologia educacional, Estudos de validação.

\begin{abstract}
Objective: To survey the main educational technologies used in the care of patients with Diabetes Mellitus. Methods: This is an integrative review of retrospective literature with descriptive-exploratory character. So, a search was performed through the descriptors "Diabetes Mellitus", "Diabetes Complications", "Educational Technology", "Validation Study", "Play and Playthings" in the following databases: LILACS, SciELO, Web of Science e PubMed, in Portuguese, English and Spanish. Results: The final sample consisted of 20 articles which it was possible to form two thematic nuclei related to the main educational technologies used in the care of diabetic patients, namely: types of care-educational technologies used to promote self-care in diabetics and interface between meaningful learning and the difficulties in implementing care-educational technologies. The current scenario of health education production uses, mainly, as a resource, digital media such as games, however it still counts on the significant participation of printed and physical tools. The need for adaptation in terms of content and language is notorious in order to meet the sociocultural demand of the public addressed. Final Considerations: The production of educational technologies for diabetics is mostly based on digital media. It was noted the lack of board games for the public in question.
\end{abstract}

Key words: Diabetes mellitus, Diabetes complications, Educational technology, Validation study.

\section{RESUMEN}

Objetivo: Realizar una encuesta bibliográfica sobre las principales tecnologías educativas que son utilizadas en el cuidado con los pacientes con Diabetes Mellitus. Métodos: Se trata de una revisión integradora de literatura retrospectiva con carácter descriptivo-exploratorio basada en los datos LILACS, Web of Science,

1Universidade Federal de Campina Grande (UFCG), Cajazeiras - PB. *E-mail: vinicius.souza15@hotmail.com ${ }^{2}$ Faculdade Santa Maria (FSM), Cajazeiras - PB.

SUBMETIDO EM: 3/2021

ACEITO EM: 3/2021

PUBLICADO EM: 5/2021 
PUBMED y SCIELO, mediante el cruce de los siguientes descriptores "Diabetes Mellitus", "Complicaciones de la Diabetes", "Tecnología Educativa", "Estudio de validación" y "Juegos y juguetes", en los idiomas portugués, inglés y español. Resultados: La muestra final fue formada por 20 artículos que emergieron en dos categorías temáticas: Tipos de tecnologías cuidado-educativas utilizadas para promover el autocuidado en diabéticos e Interfaz entre aprendizajes significativas y las dificultades en la implementación de tecnologías cuidado-educativas. El escenario actual de producción de educación para la salud utiliza, principalmente, como recurso, medios digitales como los juegos, pero aún cuenta con la participación significativa de herramientas impresas y físicas. Es notoria la necesidad de adaptación en relación a contenidos y lenguajes para atender la demanda sociocultural del público al que se dirige. Consideraciones Finales: La producción de tecnologías educativas para diabéticos se basa principalmente en medios digitales. Se señaló la falta de juegos de mesa para el público en cuestión.

Palabras clave: Diabetes mellitus, Complicaciones de la diabetes, Tecnologia educacional, Estudios de validación.

\section{INTRODUÇÃO}

O Diabetes Mellitus (DM) é caracterizada como uma das principais doenças crônicas, sendo reconhecida pela Organização Mundial de Saúde como um grave distúrbio de saúde pública. Estima-se que 463 milhões de indivíduos sejam diabéticos, esse número pode aumentar para 578 milhões em 2030 e 700 milhões em 2045. Em termos econômicos, essa doença provoca um gasto de US\$ 760 bilhões, representando cerca de 10\% dos gastos globais em saúde (IDF, 2019).

O DM consiste em um grupo heterogêneo de desordens metabólicas que se caracteriza pelo estado de hiperglicemia persistente, gerado a partir do déficit na secreção de insulina ou ineficiência da sua ação a nível celular, ou quando ambos os mecanismos estão presentes. A classificação do DM é baseada segundo princípios fisiopatológicos, sendo determinante na escolha do tratamento mais adequado. Os principais subtipos diagnosticados são o diabetes mellitus tipo 1 , o diabetes mellitus tipo 2 , o diabetes mellitus gestacional e outros tipos específicos. Os fatores causais dos principais tipos de DM (genéticos, biológicos e ambientais) ainda não são completamente conhecidos, mas o sedentarismo, consumo inadequado de alimentos, tabagismo e etnia são fatores de risco relevantes para o desencadeamento da doença (VÊSCOVI SJB, et al., 2017).

Os altos níveis de glicose sanguínea expõem os pacientes a um alto risco de desenvolvimento de complicações micro e macrovasculares a curto e longo prazo, como retinopatia, problemas cardiovasculares, nefropatia e neuropatia periférica. Tais complicações reduzem consideravelmente a qualidade de vida dos pacientes, levando à incapacidade ou até mesmo a óbito, gerando também impactos econômicos para os serviços hospitalares (VÊSCOVI SJB, et al., 2017).

Por isso, observa-se cada vez mais nas práticas em saúde a necessidade em proporcionar ações que desenvolvam nos pacientes com DM a capacidade de autogerir seu cuidado, conferindo-lhes autonomia sobre sua saúde e resultando no aumento da qualidade de vida. A partir dessa concepção, a educação em saúde aparece como uma estratégia eficaz no manejo de condições crônicas como a DM. O emprego de ferramentas tecnológicas nesse contexto é importante por auxiliar no processo de ensino e aprendizagem entre pacientes e profissionais de saúde (ALMEIDA CA, et al., 2019).

Nesse aspecto, as intervenções em saúde baseadas no uso das tecnologias educacionais mostram-se como alternativas promissoras para melhorar o autocuidado dos portadores de DM. As vantagens de aplicar tais recursos vão desde o fator lúdico à versatilidade e adaptabilidade para diversos cenários, colaborando para um maior envolvimento dos indivíduos em seu tratamento. Manutenção dos níveis glicêmicos em valores adequados, prevenção de complicações e aumento na produtividade são alguns dos benefícios alcançados com a ajuda dessas ferramentas, levando a redução dos índices de internação em decorrência dos agravos clínicos da DM (GALDINO YLS, et al., 2019).

Diante do exposto, a presente investigação realizou um levantamento bibliográfico sobre as principais tecnologias educacionais utilizadas no cuidado dos pacientes com Diabetes Mellitus. Nesse tocante, buscou- 
se demostrar o impacto positivo do uso dessas ferramentas e incentivar as equipes multidisciplinares, pesquisadores e acadêmicos da área da saúde, a inserirem recursos recreativos e didáticos validados em suas ações com os indivíduos diabéticos.

\section{MÉTODOS}

Trata-se de uma revisão integrativa de literatura retrospectiva de cunho descritivo-exploratório que realiza uma síntese dos materiais científicos existentes e proporciona o espaço para sua análise crítica. Através dela é possível reunir os conhecimentos produzidos sobre determinado tema e subsidiar as decisões práticas a serem tomadas, com base nos materiais, caracterizando a prática baseada em evidências (PBE) (SOUZA MT, et al., 2010).

Para elaboração desta revisão foram seguidas seis etapas distintas e sequenciais preconizadas na literatura: 1) definição do tema e questão de pesquisa; 2) estabelecimento de critérios de inclusão e exclusão; 3) definição das informações a serem extraídas dos estudos selecionados; 4) avaliação dos estudos incluídos na revisão; 5) análise e interpretação dos resultados e 6) apresentação da revisão/síntese do conhecimento (SOUSA LMM, et al., 2017).

Para definição da questão de pesquisa, utilizou-se a estratégia PICO, composta de quatro elementos indispensáveis para que a pergunta de pesquisa, suporte à busca bibliográfica: $\mathrm{P}$ (população ou problema); I (intervenção proposta); $\mathrm{C}$ (controle ou comparação), porém não cabe para este estudo; $\mathrm{O}$ (desfecho, resultado esperado) (STONE PW, 2002). Dessa forma, a questão norteadora foi: Quais as tecnologias educacionais e/ou estudos de validação produzidos de 2015 a 2020 sobre o cuidado com o paciente diabético?

Para responder esta questão, foi realizada uma pesquisa nas seguintes bases de dados: Literatura LatinoAmericana e do Caribe em Ciências da Saúde (LILACS), ScientificElectronic Library Online (SciELO), Web of Science, National Library of Medicine (PubMed), no mês de junho de 2020, utilizando sempre o operador booleano AND para o cruzamento em pares dos seguintes Descritores em Ciências da Saúde (DeCS): "Diabetes Mellitus", "Complicações do Diabetes", "Tecnologia Educacional", "Estudo de Validação" e "Jogos e Brinquedos", para o LILACS. Nas bases SciELO, PubMed e Web of Science foram escolhidos os descritores controlados do vocabulário MeSH - Medical SubjectHeadings, na língua inglesa: "Diabetes Mellitus", "Diabetes Complications", "Educational Technology", "ValidationStudy", "Play andPlaythings". Os quais foram utilizados em pares com o operador booleano "AND".

Como critérios de inclusão dos estudos, optou-se por artigos disponíveis na íntegra e de forma gratuita, nos idiomas português, inglês ou espanhol, publicados no período de 2015 a 2020. Os critérios de exclusão foram: revisões de qualquer estilo, artigos que não abordassem o tema da pesquisa ou que não respondessem à questão norteadora, estudos em formato de editorial e artigos duplicados em mais de uma base de dados (Tabela 1).

Tabela 1 - Publicações encontradas entre os anos de 2015 a 2020 segundo as bases de dados LILACS, SciELO, Web of Science e PUBMED.

\begin{tabular}{ccccc}
\hline Descritores & LILACS & SCIELO & Web of Science & PUBMED \\
\hline Diabetes Mellitus AND Tecnologia educacional & 12 & 11 & 27 & 143 \\
Complicações do Diabetes AND Tecnologia & 5 & 0 & 28 & 44 \\
educacional & 68 & 64 & 779 & 313 \\
$\begin{array}{c}\text { Diabetes Mellitus AND Estudo de Validação } \\
\text { Complicações do Diabetes AND Estudo de } \\
\text { validação }\end{array}$ & 15 & 5 & 221 & 130 \\
$\begin{array}{c}\text { Diabetes Mellitus AND Jogos e Brinquedos } \\
\text { Complicações do Diabetes AND Jogos e } \\
\text { Brinquedos }\end{array}$ & 1 & 0 & 0 & 10 \\
\end{tabular}

Fonte: Souza JV, et al., 2020. 
Após o procedimento da busca eletrônica, as publicações foram pré-selecionadas, inicialmente pela leitura do título e depois com base no resumo. Após a leitura na íntegra dos artigos previamente selecionados, foram identificados os artigos que compuseram a amostra final desta revisão integrativa. No total foram encontrados 1.878 estudos e selecionados 20 artigos, das seguintes bases de dados: 3 LILACS, 3 SCIELO, 10 PUBMED e 6 Web of Science.

A Figura 1 apresenta o fluxograma referente ao processo de seleção dos artigos que fizeram parte da amostra final desta seleção.

Figura 1 - Fluxograma representativo do processo de seleção dos artigos.

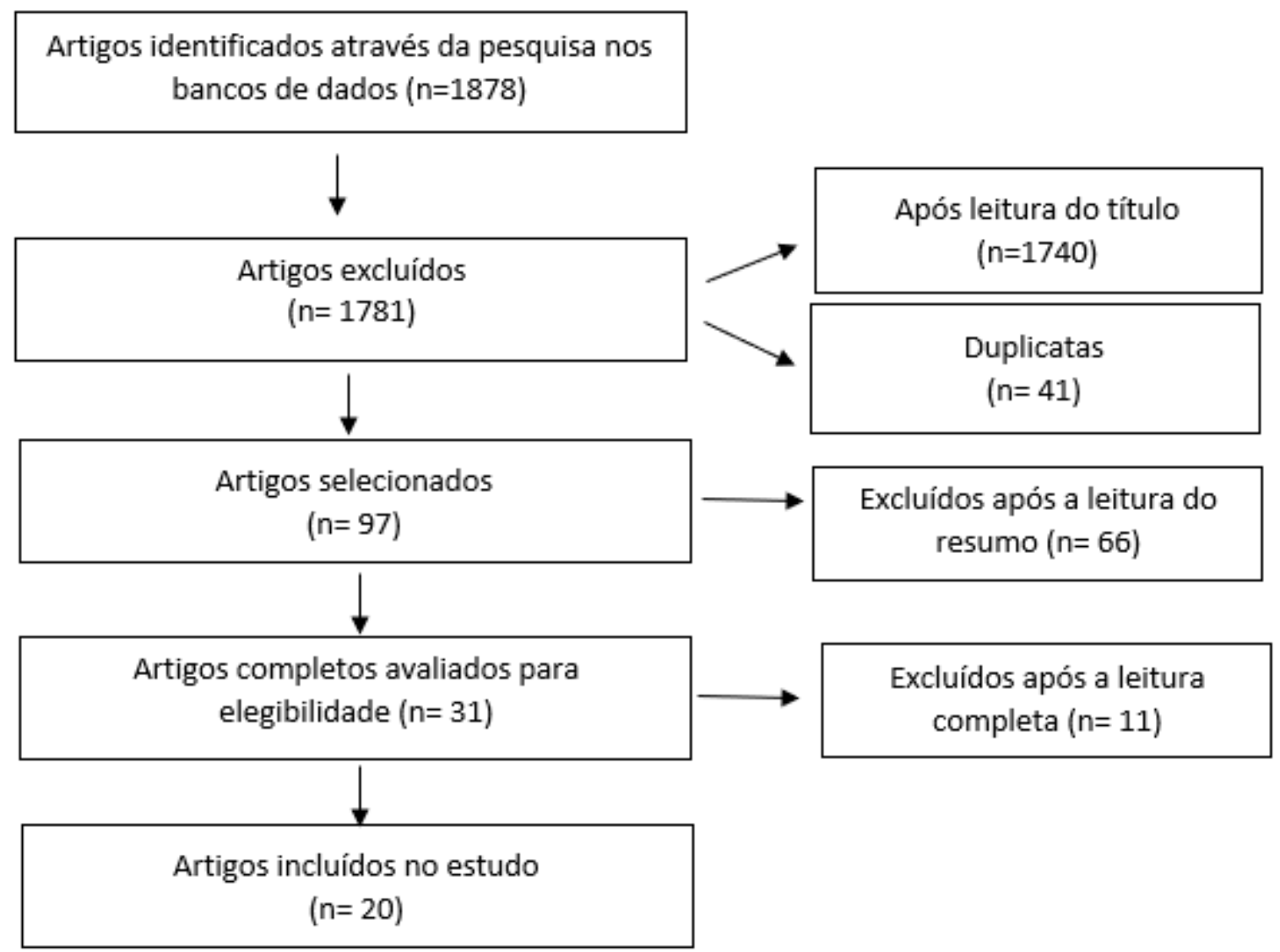

Fonte: Souza JV, et al., 2020.

Para a coleta de dados, a análise do artigo na íntegra ocorreu de forma descritiva, com intuito de reunir conhecimentos acerca do tema abordado na revisão, para isto foi preenchido um formulário de coleta de dados de acordo com o modelo previamente validado, que engloba a identificação do artigo e características metodológicas (SOUZA MT, et al., 2010).

\section{RESULTADOS}

A análise dos 20 artigos selecionados viabilizou a formação de dois núcleos temáticos relacionados às principais tecnologias educacionais utilizadas no cuidado com pacientes diabéticos: Tipos de tecnologias cuidativo-educacionais utilizadas na promoção do autocuidado em diabéticos e Interface entre aprendizagem significativa e as dificuldades na implementação das tecnologias cuidativo-educacionais. O Quadro 1 sintetiza as informações disponibilizadas pelos artigos que foram incluídos na revisão de literatura. 
Quadro 1 - Apresentação das características dos artigos incluídos na Revisão Integrativa.

\begin{tabular}{|c|c|c|}
\hline $\mathbf{N}$ & Autores (Ano) & Principais achados \\
\hline 1 & WANG J, et al. (2015) & $\begin{array}{l}\text { O "Diab", um jogo de imersão de realidade, é passível de adaptação cultural e eficaz na motivação de mudanças } \\
\text { comportamentais dietéticas e prática de atividades físicas em crianças de Hong Kong. }\end{array}$ \\
\hline 2 & CALLE-BUSTOS AM, et al. (2017) & $\begin{array}{l}\text { O jogo de realidade aumentada, baseado na apresentação de alimentos ricos em carboidratos projetados em } \\
\text { pratos reais, mostrou-se útil na transmissão de conhecimento acerca dos carboidratos em crianças com diabetes. }\end{array}$ \\
\hline 3 & KLAASSEN R, et al. (2018) & $\begin{array}{l}\text { A plataforma educacional, motivacional e de monitoramento para pacientes jovens com diabetes teve resistência } \\
\text { ao uso pelos usuários, revelando a necessidade de melhorias para aplicação no público em questão. }\end{array}$ \\
\hline 4 & MOURA DJM, et al. (2017) & $\begin{array}{l}\text { A construção da cartilha propõe favorecer o empoderamento e promover a saúde de crianças com DM. Este tipo } \\
\text { de tecnologia funciona como suporte a profissionais, crianças e famílias, para que superem dificuldades e } \\
\text { esclareçam dúvidas. }\end{array}$ \\
\hline 5 & SOARES GM, et al. (2018) & $\begin{array}{l}\text { O livreto de jogos educativos acerca de doenças metabólicas, obesidade e diabetes é aplicável na disseminação } \\
\text { do conhecimento da temática entre estudantes do ensino médio. }\end{array}$ \\
\hline 6 & OWOLABI EO, et al. (2020) & $\begin{array}{l}\text { O uso de mensagens de texto como ferramenta educativa entre os pacientes diabéticos em questão não mostrou } \\
\text { resultados significativos na adesão a medidas positivas dietéticas e de prática de atividades físicas. }\end{array}$ \\
\hline 7 & PENNAFORT VPS, et al. (2018) & $\begin{array}{l}\text { A utilização de brinquedos terapêuticos instrucionais logra efeitos positivos na convivência da criança com a } \\
\text { terapêutica do DM tipo } 1 \text {, sanando dúvidas e promovendo o autocuidado de forma humanizada. }\end{array}$ \\
\hline 8 & SERAFIM ARRM, et al. (2019) & $\begin{array}{l}\text { O teste de usabilidade teve validação positiva e as sugestões contribuíram para melhoria da qualidade das } \\
\text { funções e interação, favorecendo o alcance das metas do jogo. }\end{array}$ \\
\hline 9 & GONÇALVES MS, et al. (2019) & $\begin{array}{l}\text { A cartilha educativa é uma proposta de trabalho para favorecer a educação nutricional de pessoas com diabetes } \\
\text { e também um instrumento pelo qual se pode despertar a vontade do leitor na ampliação do seu potencial de } \\
\text { autocuidado. }\end{array}$ \\
\hline 10 & SILVA JP, et al. (2018) & $\begin{array}{l}\text { O simulador de baixo custo para aplicação de insulina pode ser adotado pelas equipes de saúde como ferramenta } \\
\text { de capacitação dos pacientes, diminuindo a lacuna entre conhecimento e prática. }\end{array}$ \\
\hline 11 & AQUINO JA, et al. (2016) & $\begin{array}{l}\text { A cartilha apresenta boas características de validação de conteúdo para ser utilizada em um programa de } \\
\text { educação para empoderamento de pacientes com DM. }\end{array}$ \\
\hline
\end{tabular}




\begin{tabular}{|c|c|c|}
\hline $\mathbf{N}$ & Autores (Ano) & Principais achados \\
\hline 12 & MARQUES ADB, et al. (2020) & $\begin{array}{l}\text { Os usuários do aplicativo apresentado acham fácil inserir e corrigir dados, acreditam que o produto atende às } \\
\text { necessidades de consumo. Definem a interface como intuitiva e interativa, bem como, a linguagem acessível. } \\
\text { Assim, concordam totalmente com todos os itens e consideram a ajuda/dica fornecida pelo App como útil. }\end{array}$ \\
\hline 13 & ROSENDO I e SANTIAGO LM (2017) & Estudo metodológico de validação \\
\hline 14 & AGUILA IP, et al. (2018) & $\begin{array}{l}\text { O uso de Tecnologias da informação e Comunicação são positivas na complementação do acompanhamento e } \\
\text { educação em saúde de pacientes adultos diabéticos. }\end{array}$ \\
\hline 15 & BAI LL, et al. (2018) & $\begin{array}{l}\text { O jogo de adivinhação, por ser uma ferramenta agradável e de fácil aplicação, mostrou-se positivo para consolidar } \\
\text { a compreensão sobre as complicações em pacientes com DM. }\end{array}$ \\
\hline 16 & ABU-SAAD K, et al. (2019) & $\begin{array}{l}\text { O software I-ACE apresentou potencial no aconselhamento alimentar e na melhoria do comportamento } \\
\text { relacionado ao estilo de vida em diabéticos pertencentes a uma população minoritária. }\end{array}$ \\
\hline 17 & NWOLISE CH, et al. (2017) & $\begin{array}{l}\text { O aplicativo de Informações sobre Preconceito e Diabetes (PADI) tem potencial no aprimoramento dos cuidados } \\
\text { pré-concepção, assim como melhorar os resultados glicêmicos em gestantes diabéticas. }\end{array}$ \\
\hline 18 & ADU MD, et al. (2020) & $\begin{array}{l}\text { A ferramenta MyCare Hub tem índices aceitáveis no processo de retenção e envolvimento dos participantes, } \\
\text { apontando uma possibilidade para modificar comportamentos e contribuir para o autogerenciamento dos } \\
\text { pacientes com DM. }\end{array}$ \\
\hline 19 & FERREIRA JSSP, et al. (2019) & $\begin{array}{l}\text { O SOPED foi validado com um alto grau de concordância entre especialistas em DM e pacientes, sendo capaz } \\
\text { de colaborar para a autogestão, automonitoramento e atendimento personalizado. }\end{array}$ \\
\hline 20 & ABAZA H e MARSCHOLLEK M (2017) & $\begin{array}{l}\text { A educação por SMS é um método exequível e aceitável que pode ajudar no controle glicêmico e na mudança de } \\
\text { comportamentos para a autogestão entre os egípcios diabéticos. }\end{array}$ \\
\hline
\end{tabular}

Fonte: Souza JV, et al., 2020. 


\section{Tipos de tecnologias cuidativo-educacionais utilizadas na promoção do autocuidado em diabéticos}

A utilização de tecnologias na educação, suporte e gerenciamento do paciente diabético conta com uma enorme heterogeneidade de intervenções e metodologias. De modo que, apresentou um acentuado crescimento após a introdução da tecnologia móvel, que oferta à sociedade a disponibilidade onipresente de informações (GREENWOOD DA, et al., 2017). Esse estudo aponta para a diversidade nas abordagens, ressaltando o amadurecimento das ações apresentadas em revisões sistemáticas a partir do ano de 2013. Nesse sentido, é pertinente a categorização dos achados de acordo com a ferramenta empregada na promoção do autocuidado e educação de pacientes diabéticos.

\section{Tecnologias educacionais baseadas em recursos digitais}

O avanço contemporâneo das tecnologias de informação e comunicação permitiu a apropriação dessas para o campo da educação em saúde, mostrando um efeito promissor graças a facilidade que oferecem na difusão de conhecimentos. De maneira aplicada, no diabetes, têm ajudado a prevenir complicações, reduzindo riscos e custos no manejo do paciente diabético (AGUILA IP, et al., 2018).

Dos estudos analisados, o desenvolvimento de games online e aplicativos para celular lideram as iniciativas de abordagem ao público diabético. Wang J, et al. (2015) relatam que os videogames são capazes de criar um ambiente virtual e fazer os usuários se sentirem imersos na nova realidade, além de serem populares e vinculados ao entretenimento. Semelhantemente, Nwolise $\mathrm{CH}$, et al. (2017) mostram os benefícios no uso de aplicativos através do desenvolvimento de mudanças comportamentais significativas em gestantes diabéticas, pelo emprego do método, além de dar ênfase à praticidade de portar consigo a ferramenta educativa.

A tecnologia móvel apresenta uma maneira fácil e eficaz para alcançar uma população maior, sendo os serviços de mensagem de texto uma alternativa prática dado o grande número de usuários de telefones móveis e ainda a compatibilidade do serviço com todos os tipos de celulares (ABAZA H e MARSCHOLLEK $M, 2017)$. Esse estudo conclui que as intervenções utilizando SMS aprimoraram notavelmente os aspectos de conhecimento acerca da doença, apresentaram baixo custo e o material educativo pode ser entregue a qualquer hora. Vale salientar que se enviados regularmente, eles também fornecem doses leves de informação que podem ser facilmente compreendidas, auxiliando no processo de adesão ao tratamento. Esse dado corrobora com os achados do estudo de Owolabi EOM, et al. (2020) que apontam para o uso dessa tecnologia no auxílio do paciente, como lembrete, a fim aumentar a adesão à medicação.

Por último, na transmissão de informações via monitores, os pacientes tiveram acesso, nas salas de espera das clínicas, a um sistema capaz de fornecer dados advindos do seu histórico médico, a fim de encorajar o autogerenciamento, conhecimentos importantes a respeito da DM e, ainda, dicas sobre alterações nos hábitos alimentares (AGUILA IP, et al., 2018). A eficácia da educação através do sistema de informação multimídia foi avaliada como positivo no controle glicêmico e perfil lipídico dos pacientes que fizeram uso da ferramenta em comparação ao grupo controle do estudo.

\section{Tecnologias educacionais baseadas em material didático impresso}

Os artigos mostraram, em menor frequência, a utilização de mídias impressas para orientação educativa acerca do diabetes. A atual priorização de métodos ativos da aprendizagem pode ser entendida pela linha de pensamento defendida por Bai LL, et al. (2018), na qual, apontam para uma maior consolidação do conhecimento através do seu uso, uma vez que rompem com o modelo de aprendizagem passiva, que impede o estabelecimento da troca de informações, e é capaz de oferecer experiência para a aplicação dos saberes nos problemas diários. Outro ponto a ser considerado é o alto custo na impressão das cartilhas educativas ou panfletos, assim como, o fato dos pacientes se sentirem desencorajados a ler e processar a carga de informações (ABAZA H e MARSCHOLLEK M, 2017).

No entanto, as cartilhas e folhetos ainda figuram um importante material de apoio, apontada como alternativa viável na instrução e sensibilização de pacientes a respeito do processo saúde-doença, dando condições ao indivíduo para a autogestão, e, ainda, adoção de hábitos de vida saudáveis (GONÇALVES MS, et al., 2019). 
Para Moura DJM, et al. (2017), a utilização de material educativo escrito e ilustrado, pela equipe de saúde, pode ser adotada quando se deseja reforçar as orientações verbalizadas. A via impressa do instrumento permite que o usuário a leve para seu domicílio, portando informações importantes sobre a temática trabalhada, o que pode impactar de forma positiva a educação do público assistido.

\section{Tecnologias educacionais pautadas em outras abordagens}

Os resultados mostraram outros instrumentos capazes de mediar a transmissão de informações aos pacientes assistidos pelos profissionais da saúde, como, por exemplo, a criação de brinquedos terapêuticos. Pennafort VPS, et al. (2018) analisaram a experiência em crianças com Diabetes Mellitus tipo 1 nos cuidados referentes a monitoração glicêmica e a aplicação de insulina por meio do brinquedo terapêutico instrucional (BTI). De modo geral, o BT1 teve por objetivo conhecer as emoções e as experiências vivenciadas pelas crianças, servindo como um instrumento que facilita a aprendizagem ativa para a realização dos procedimentos terapêuticos. Através das simulações dos cuidados no controle glicêmico com o BTI, notou-se que esse recurso foi útil na comunicação efetiva dos cuidados essenciais no controle glicêmico, desenvolvendo suas habilidades de autocuidado. Logo, essa abordagem educativa contribui para reduzir o estresse dos procedimentos terapêuticos, sensibilizar, empoderar e modificar a vivência da criança no enfrentamento dos problemas inerentes a sua condição.

Silva JP, et al. (2018), levantam a relevância da utilização de simuladores em adultos. A implementação desses dispositivos se justifica pela carência de ações que tangenciem de melhor maneira as demandas do dia a dia. Nesse sentido, o uso de bonecos com locais apropriados para a aplicação da insulina é capaz de oferecer uma experiência onde as peculiaridades de uma situação real são ofertadas. O simulador recorre a criação de um ambiente artificial para avaliar, testar, praticar e desenvolver as habilidades dos pacientes, bem como dos cuidadores, culminando na prevenção de agravos e seguridade do melhor manejo.

Ademais, ressalta-se a existência do uso de jogos em grupos. A realização de dinâmicas entre os pacientes favorece o fortalecimento das habilidades sociais, bem como, cria um vínculo, por meio das interações, capaz de ser lido pelo sujeito como rede de apoio. Desse modo, é elencado em um dos estudos a utilização do Jogo da Adivinhação, emergindo como alternativa divertida e de fácil execução para a compreensão das complicações do diabetes por recém-diagnosticados (BAI LL, et al., 2018).

\section{Interface entre aprendizagem significativa e as dificuldades na implementação das tecnologias cuidativo-educacionais}

Os estudos analisados mostram que a consolidação do conhecimento é expressa como aprendizagem significativa, quando a informação é empregada na vida diária do indivíduo (MOURA DJM, et al., 2017). A partir disso, aliar aspectos lúdicos ao cognitivo, através de metodologias ativas, se torna uma tática fundamental para o processo de ensino-aprendizagem.

O rompimento com o modelo de ensino mecânico ou memorístico é levantado como alternativa inovadora na produção de educação em saúde. A proposta integra os conhecimentos prévios do sujeito e tornando-o ativo na construção do saber. Nesse sentido, é importante fundamentar a abordagem em estratégias que passem pela via da interatividade e motivação, provocando o indivíduo e inserindo as informações de saúde na sua rotina (SERAFIM ARRM, et al., 2019).

Klaassen $\mathrm{R}$, et al. (2018) sugerem a ludificação da problemática como ferramenta didática. O estudo mostrou que a utilização de games, estes são capazes de condensar elementos gráficos que cooperam com a identificação, estimula a imaginação, além de integrar uma narrativa eficiente na transmissão de informações, contendo desafios e regras que fornecem, de maneira divertida, instrumentos necessários para a execução do autogerenciamento.

Nesse tocante, o feedback do emprego dessas tecnologias necessita de um indicador que vá além da avaliação da taxa de uso. Para a análise da retenção do conteúdo transmitido, é fundamental a verificação do envolvimento do usuário, bem como, sua intenção no uso continuo do saber adquirido, que implica na mudança comportamental (ADU MD, et al., 2020). A exemplo, Calle-Bustos AM, et al. (2017) avaliaram a 
receptividade de um jogo, voltado para o público infantil, em que eram apresentadas e reforçadas as opções de carboidratos para crianças diabéticas. O sucesso acolhimento da ferramenta pôde ser verificado em um questionário aplicado onde obteve-se uma média de 4,58, em uma escala de 1 a 5 , ao questionar os usuários se gostariam de usar esses jogos para aprender mais sobre o diabetes.

Entretanto, há autores que lançam mão de métodos mais tradicionais como elo de aproximação com a comunidade em questão, defendendo a obtenção de resultados positivos na aplicação destes. A utilização de materiais como folders, manuais, folhetos, cartilhas e livretos, por exemplo, pode ser um recurso eficiente e aplicável na transmissão de informação e motivação pelos profissionais da saúde. Estes dispositivos facilitam a comunicação, fomentam a adesão ao tratamento e estimulam a autonomia do sujeito (GONÇALVES MS, et al., 2019).

Contudo, para a efetivação da educação em saúde algumas barreiras precisam ser transpostas, de maneira a visualizar os usuários de forma integral, assumindo a singularidade dos sujeitos envolvidos e a multiplicidade de questões que interferem nos seus hábitos de vida. Abu-Saad K, et al. (2019) levantam alguns desafios, sobretudo para as minorias, referentes às disparidades socioeconômicas, étnico/raciais e culturais. Nessa perspectiva, sugerem o uso da tecnologia da informação como meio gerador de novas oportunidades para contemplar as necessidades individuais e gerar medidas de aconselhamento e instrução personalizadas.

Partindo dessa linha, um estudo mostrou a adesão à dieta saudável por pacientes com DM em uma comunidade rural na África do Sul, justificando a não-adesão dos pacientes avaliados pela desigualdade socioeconômica (OWOLABI EOM, et al., 2020). Desse modo, a fim de executar uma abordagem centrada no paciente, as ferramentas utilizadas nas intervenções podem ser adaptadas de acordo com as necessidades da população. No mesmo estudo, há ainda a proposição da oferta de informações pertinentes à realidade cultural que o indivíduo está inserido, no caso, a demonstração de maneiras de adotar hábitos alimentares saudáveis a partir do repertório de alimentos disponíveis.

Além disso, outro aspecto importante é levantado quando se trata do emprego de tecnologias na promoção da saúde: o comprometimento individual. O envolvimento na atividade proposta, e ainda, o acolhimento do que é novo para a prática diária, passa necessariamente pelo desejo do paciente, envolvendo questões de sua personalidade. Logo, é essencial a pré-disposição e abertura do participante para a afirmação da interação necessária e êxito da intervenção. Soares GM, et al. (2018) ressaltam a problemática ao analisar o emprego de um livreto interativo sobre doenças metabólicas em estudantes do ensino médio.

Ademais, destaca-se a importância da adaptação da interface do instrumento empregado na realização das ações. Nesse sentido, é salientado o ajuste do método de comunicação ao público trabalhado, expressa por meio da linguagem e aspectos lúdicos empreendidos. Klaassen R, et al. (2018) ilustram a adequação como elemento imprescindível ao obter, como resultado de seu estudo, a resistência ao uso de uma plataforma virtual educacional voltada para jovens diabéticos. Estes, apontaram a ferramenta como inadequada para a idade do grupo, dados alguns elementos infantis da interface, além de levantarem pontos que dizem respeito à simplificação e objetivação do conteúdo apresentado.

\section{CONSIDERAÇÕES FINAIS}

Foi possível identificar, nesta revisão integrativa, as tecnologias que estão sendo utilizadas para a educação em saúde do paciente diabético, bem como, as dificuldades a serem superadas pelo uso dessas. Com isso, a partir do presente estudo, foi possível verificar que existe uma lacuna na produção e aplicação das tecnologias cuidativas com enfoque no público diabético. Não foram levantados estudos de validação, ou relato de uso, de alguns jogos físicos, a exemplo: jogos de tabuleiro, construídos para o paciente com DM. Nesse sentido, se fazem pertinentes a criação e investigação da usabilidade desse instrumento, uma vez que reúne características atrativas, concernentes ao lúdico e, ainda, passíveis de adaptação para os mais diversos públicos, a fim da transmissão do conhecimento. 


\section{REFERÊNCIAS}

1. ABAZA H, MARSCHOLLEK M. SMS education for the promotion of diabetes self-management in low \& middle income countries: a pilot randomized controlled trial in Egypt. BMC Public Health, 2017; 17(1): 962.

2. ABU-SAAD K, et al. Development and Efficacy of an Electronic, Culturally Adapted Lifestyle Counseling Tool for Improving Diabetes-Related Dietary Knowledge: Randomized Controlled Trial Among Ethnic Minority Adults with Type 2 Diabetes Mellitus, J Med Internet Res 2019; 21(10): e1367.

3. ADU MD, et al. User Retention and Engagement with a Mobile App Intervention to Support Self-Management in Australians With Type 1 or Type 2 Diabetes (My Care Hub): Mixed Methods Study JMIR Mhealth Uhealth 2020; 8(6): e17802.

4. AGUILA IP, et al. Multimedia education to support management of type 2 diabetes patients. A quasi-experimental study [La educación multimedia como apoyo en el manejo de pacientes con diabetes tipo 2. Estudio cuasi experimental]. Cir Cir, 2018; 86(5).

5. ALMEIDA CA, et al. Avaliação de um ambiente virtual de aprendizagem sobre ações educativas para pessoas com diabetes mellitus. Escola Anna Nery, 2019; 23(4).

6. AQUINO JA, et al. Cartilha educativa sobre diabetes: elaboração e validação de conteúdo, Semina: Ciências Biológicas e da Saúde, 2016; 37(1): 77-82.

7. BAI LL, et al. Using guessing game to increase complication awareness of patients with newly diagnosed type 2 diabetes. Acta endocrinologica (Bucharest), 2018; 14(3): 401-407.

8. CALLE-BUSTOS AM, et al. An augmented reality game to support therapeutic education for children with diabetes. PLoS One, 2017; 12(9): e0184645.

9. FERREIRA JSSP, et al. Rehabilitation technology for self-care: Customised foot and ankle exercise software for people with diabetes. PLOS ONE, 2019 14(6): e0218560.

10. GALDINO YLS, et al. Validation of a booklet on self-care with the diabetic foot. Revista brasileira de enfermagem, 2019; 72(3):780-787.

11. GONÇALVES MS, et al. Construção e validação de cartilha educativa para promoção da alimentação saudável entre pacientes diabéticos. Rev. bras. promoç. Saúde, 2019; 32, 1-9.

12. GREENWOOD DA, et al. A Systematic Review of Reviews Evaluating Technology-Enabled Diabetes SelfManagement Education and Support. Journal of Diabetes Science and Technology, 2017; 11(5):1015-1027.

13. INTERNATIONAL DIABETES FEDERATION. IDF Diabetes Atlas. 9th ed. Brussels, Belgium: International Diabetes Federation; 2019.

14. KLAASSEN R, et al. Design and Evaluation of a Pervasive Coaching and Gamification Platform for Young Diabetes Patients. Sensors (Basel), 2018; 18(2): 402.

15. MARQUES ADB, et al. Usabilidade de um aplicativo móvel sobre o autocuidado com o pé diabético. Rev. Bras. Enferm., 2020; 73(4): e20180862.

16. MOURA DJM, et al. Development of a booklet on insulin therapy for children with diabetes mellitus type 1 . Rev Bras Enferm, 2017; 70(1): 3-10.

17. NWOLISE CH, et al. Exploring the acceptability and feasibility of a preconception and diabetes information app for women with pregestational diabetes: A mixed-methods study protocol. Digit Health, 2017; 3: 2055207617726418.

18. OWOLABI EOM, et al. Impact of mobile phone text messaging intervention on adherence among patients with diabetes in a rural setting. Medicine, 2020; 99(12): e18953.

19. PENNAFORT VPS, et al. Instructional therapeutic toy in the culture care of the child with diabetes type 1. Rev Bras Enferm, 2018; 71(3): 1334-42.

20. ROSENDO I, SANTIAGO LM. Validação de três folhetos informativos sobre diabetes, sua terapêutica e exercício físico. Rev Port Med Geral Fam, 2017; 33(4): 244-250.

21. SERAFIM ARRM, et al. Construção de serious games para adolescentes com diabetes mellitus tipo 1. Acta paul. Enferm,2019; 32(4):374-381.

22. SILVA JP, et al. Construção e validação de simulador de baixo custo para capacitação de pacientes com diabetes mellitus e/ou de seus cuidadores na aplicação de insulina. Esc. Anna Nery, 2018; 22(3): e20170387.

23. SOARES GM, et al. Impact of a playful booklet about diabetes and obesity on high school students in Campinas, Brazil. Adv Physiol Educ. 2019; 43(3): 266-269.

24. SOUSA LMM, et. al. A metodologia de revisão integrativa da literatura em enfermagem. Revista Investigação em Enfermagem, 2017; 2:17-26.

25. SOUZA MT, et al. Revisão integrativa: o que é e como fazer. Einstein (São Paulo), 2010; 8(1):102-106.

26. STONE PW. Popping the (PICO) question in research and evidence-based practice. Appl Nurs Res, 2002; 15(3).

27. VÊSCOVI SJB, et al. Aplicativo móvel para avaliação dos pés de pessoas com diabetes mellitus. Acta Paulista de Enfermagem, 2017; 30(6):607-613.

28. WANG J, et al. Acceptability and Applicability of an American Health Videogame with Story for Childhood Obesity Prevention Among Hong Kong Chinese Children. Games Health J. 2015; 4(6): 513-519. 